\title{
el revestimiento refractario en la industria del cemento
}

ANTONIO SARABIA

Ingeniero Industrial. Diractor Thenjico de Sosiedad A. E. Cementos Portland

Conferencia pronunciada en Barcelona, el pagata mes de novjembre, eon motivo de fa 11 Rtunión Técnica de la Secesón de
Espantola de Cerámica.

Un horno para cemento es, en esencia, una entidad realizada con el fin de que, en su seno, se desarrolle un pro ceso de transformación sobre materiales de naturaleza y preparación adecuadas para conseguir que los oxidos de calcio, silicio, aluminio y hierro, por ellos aportados, adquieran una nueva disposición estructural que implica la aparición de nuevos compuestos químicos, cristalizados en el seno de un vidrio fundido. El producto obtenido, universalmente conocido por clínker de cemento Portiand (refiriéndose al más caracteristico), es el resultado de la "congelacín"s, por enfriamfento rápido en el horno mismo, del sistema nuevo en equilibrio a ia alta temperatura de su prođucción.

Todo proceso de transformación material supone la realización de otro energético, en el que cierta cantidad de energía experimenta un cambio formal.

En el horno para cemento, en la fase específica del proceso, paso de oxidos presentes a clinker, se requiere la evolución de energia térmica a quimica e inversamente. Esta evolución sólo se consigue a temperatura del orden de $1.500^{\circ} \mathrm{C}$, variable según la composición y propiedades de las sustancias de partida y eșa afirmación da caracter, en cierto sentido, al horno: En alguna región del mismo, la masa en reacción ha de alcanzar, de modo ineludible, una tem. peratura de acuel orden para tlegar a ser aclínker».

Toda entidad que ha de realizar una función especifica, ha de estar diferenciada del ámbito en que esta situada. Por tanto, el horno para cemento, séame permitida la palabra, ha de tener upieln y de ella voy a ocuparme ante vuestra amable atención, ya que vuestro interes de ceramistas y el mio de cementero convergen, por lo menos, en una de las partes de aquélla.

En el intento de hacer más clara mi exposición, voy a servirme de la información que ofrece wn horno indus. trial para cemento, en régimen, a través de los sradientes térmicos, considerados a lo largo del homo, del material en reacción en la cara interior de su upieln y de los gases que lo recorren como vehfculo de la energía a disposición del proceso, si aquélla se suministra por combustión del carbón o cualquier otro combustible liquido o gaseoso. La figura numero 1, publicada por M. A. Folliot (1), reproduce el curso de los gradientes referidos, en un horno rotatorio, para cemento, via whímeda:

La linea correspondiente al material en reacción, en lineas generales, caracteriza tramos de horno en los que se realizan distintos procesos y que en el léxieo cementero se designan como:

\section{Z O N A D E}

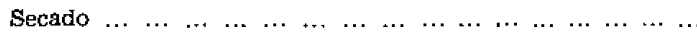
Deshidratación (y calefacción preliminar) $\ldots \ldots \ldots \ldots$

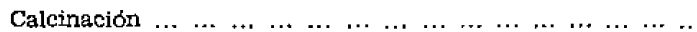

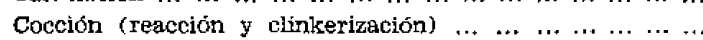

Enfriamiento (prolongado fuera del horno en el enfriador).
TEMPERATLIRAS

Entre $20 \mathrm{y} \quad 100^{\circ} \mathrm{C}$

n $\quad 100 \mathrm{y} \quad 600^{\circ} \mathrm{C}$

i) $600 \mathrm{y} \quad 950^{\circ} \mathrm{C}$

b $950 \mathrm{y} 1.450^{\circ} \mathrm{C}$

1.450 y $1.100^{\circ} \mathrm{C}$

En esas designaciones se engloban procesos complejos, cuya descripción no creo interesante en este" momento. Solamente quiero llamar vuestra atención sobre la región del horno que he designado como zona de cocción. pues en ella, por razón de la alta temperatura requerida, por la aparición de fase liquida que hace posible la realizacton en ella, por razon de la alta temperatura requerida, por la aparicion de fase liquida que hace posioje la realizacion caracteristica del
el rẹsto del horno. 
I. régimen 200 t/día. - II. régimen 100 t/día

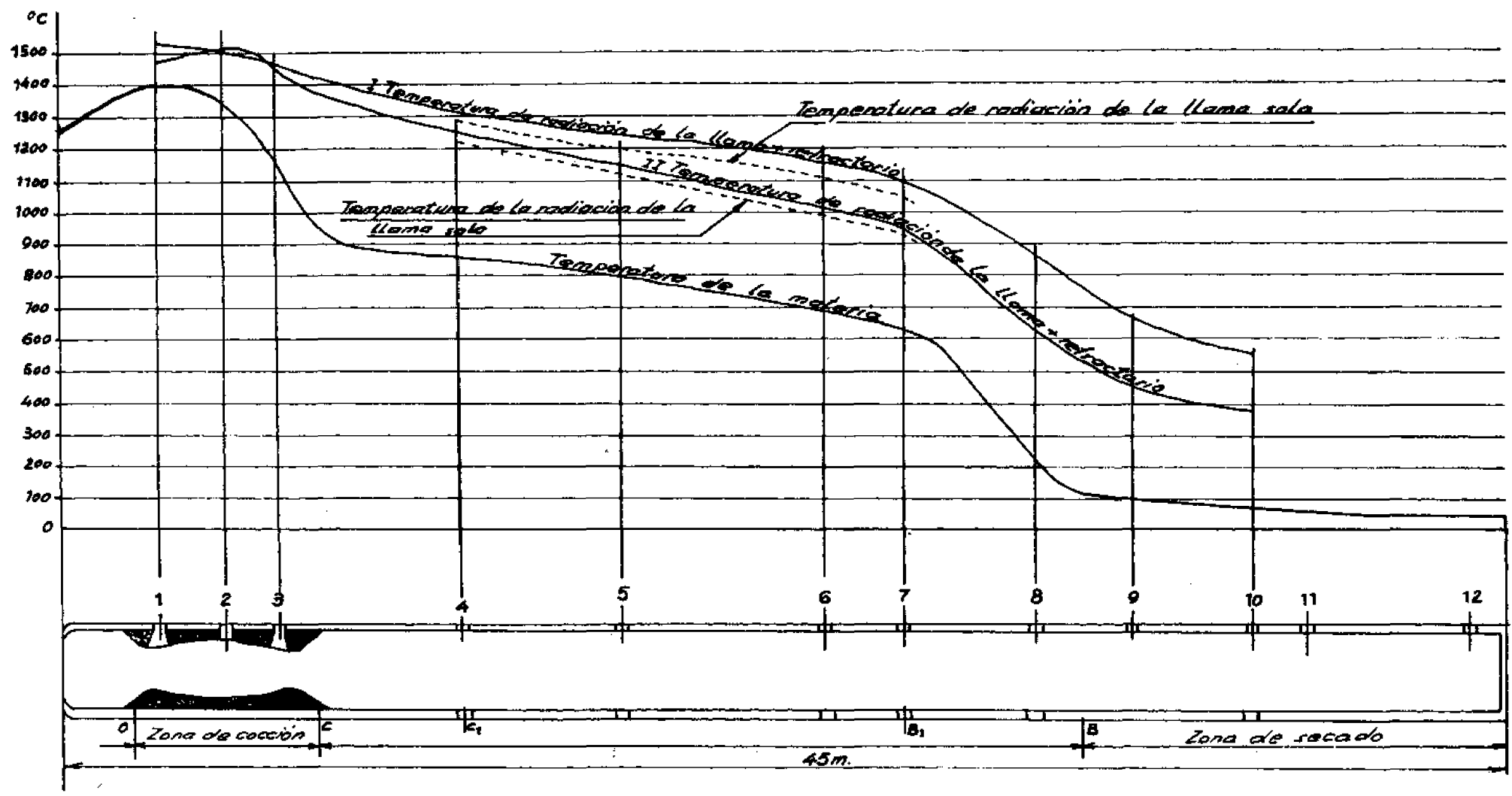

Fig. 1.-Gradionte de temperatura a to largo de un horno para oemento. 


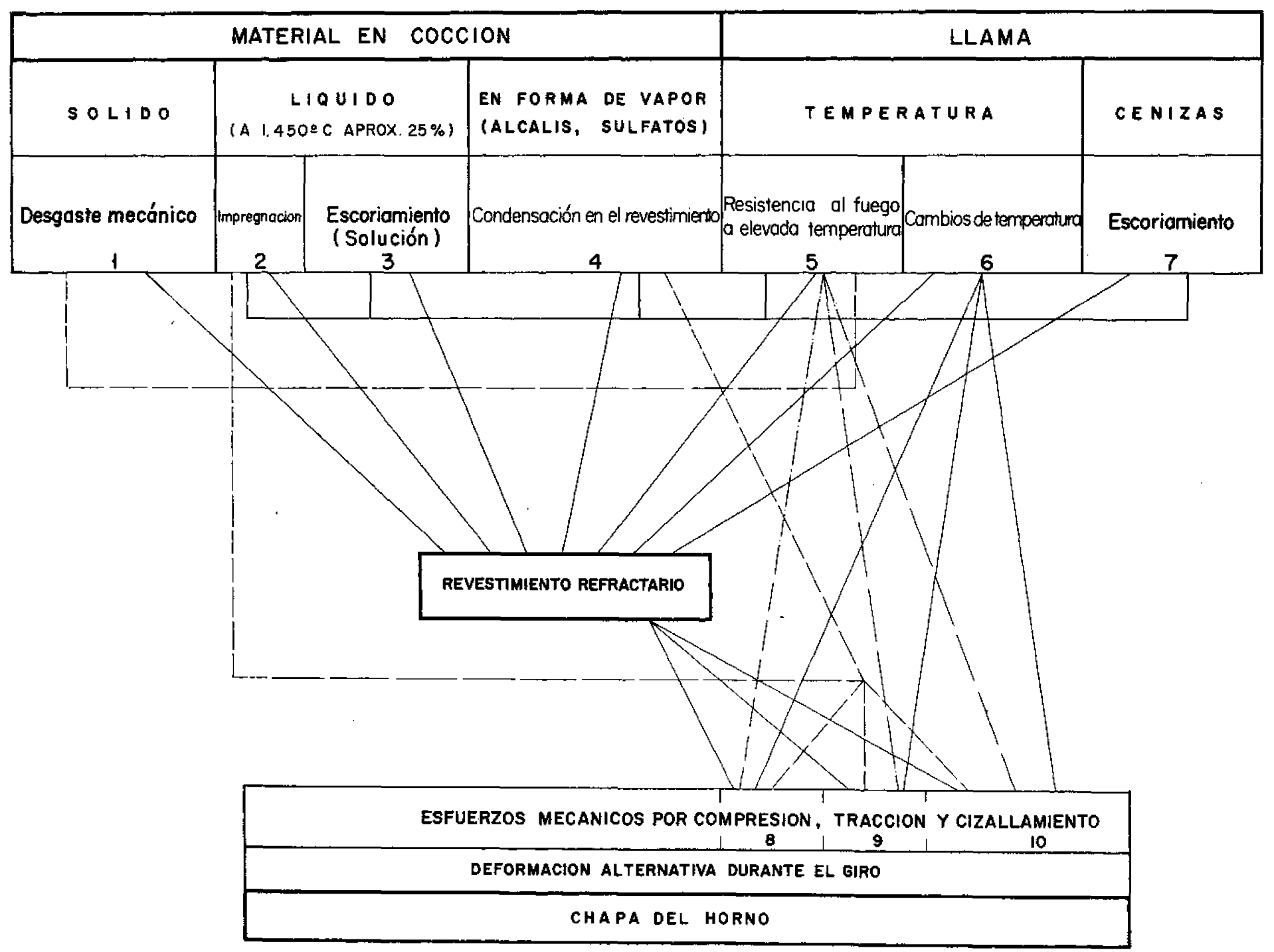

Fiž. 2.-Foliestacioner sobre la apiels del horcu de cemento. 
La zona de cocción, rodeando la llama del mechero de combustión, es, por decir asi, el scrisoln o waboratorio» del horno rotatorio para cemento. En el vertical, las condiciones de marcha del proceso establecen una zona analoga con las mismas exigencias.

E1 resto del horno sirve para realizar operaciones previas al proceso de clinkerización, secado, descomposición de minerales arcillosos y descarbonatación de la caliza, principalmente.

En la tecnologia moderna de producción de cemento, aunque formando wnidad con un tubo rotatorio, aquellas operactones previas tienen lugar a veces en dispositivos externos a aquél (Intercambiadores Humboidt, Parrilla Lepol, Recuperadores Miag, Smidth, etc.).

Aunque no muy extendidas, o en periodo de estudio de aplicación, existen tecnologías de producción del cemento, en las que el horno $n 1$ es el rotatorio $n^{\dagger}$ es el vertical clástco (Parrilla Lurgi, proceso Picel, cemento fundido al horno eléctrico, etc.).

Si a la observación del gradiente de material a lo largo del homo, sumamos la del gradiente de la cara inter na del mismo, podemos adelantar una conclusión importante: En el horno rotatorio, el paramento interior de in "upiely del horno se halla sometido a variaciones de temperatura de frecuencia del orden de una vez al minuto y de una cuantfa relativamente elevada, aunque quizés un poco inferior que la que se deduce de la figura 1, por razón del método ernpleado por Folliot para definirla.

La upieln del borno rotatorio de cemento $y$, también, la del vertical, se compone de:

1. El cllindro de chapa.

2." El material refractario.

3. La acostra) o wincrustación (en la región en que se produce, precisamente la que hemos llamado de ucoc ciónss).

Estimo que los tres elementos son interdependientes, de modo inevitable.

El fallo de cualquiera de ellos implica el fallo de los demás. Su correcto funcionamtento, como unitad, representa para el horno la actividad ininterrumpida por largas temporadas.

Conjuntamente, chapa, refractario y ucostran, han de hacer frente a las solicitaciones originadas por la realización del proceso mismo. Conseguir un comportamiento eflcaz del sistema, representa un factor importante en la economía del proceso cementerò.

La figura 2, tomada de un trabajo de A. Majdic y H. E. Schwiete (2), resume, de modo gráfico, las solicftacio nes aludidas, su origen y las partes de la upieln del horno que han de ser capaces de absorberlas.

La llama del mechero de combustion influye söbre la ucostran y el refractario por razón de las variactones de su temperatura y por su contenido en cenizas, si el combustible es carbón. También sobre la chapa, a través de las variaciones experimentadas por el refractario en contacto con ella.

El material que avanza a lo largo del horno origina:

Por sus partes solfdas, desgaste del refractario por rozamiento, más acentuado en la zona de enfriamlento por la dureza del clínker.

Por su parte líquida $\left(25 \%\right.$ a $1.450^{\circ} \mathrm{C}$ ), imbibición y escorificación del refractario, equivalente a decir agresión quí mica de aquél.

Por su parte gaseosa (sublimación de álcalis, en las zonas más calientes) condensaclones que dan lugar a modi. ficaciones en la región del refractario, en contacto con el material, que se difunden en aquél.

Cada punto de la "pieln del horno rotatorio sufre-como ya dijimos-una vez por minuto, aproximadamente, la acción de un cambio de temperatura del orden de $200^{\circ} \mathrm{C}$. En su movimiento rotatorio acumula calor radiado por la llama del mechero, para cederlo al material que rueda sobre ella.

Vuelvo a insistir en que el rozamiento, la agresion quimica, las condensactones, etc., directamente sufridos por el refractario, a por el refractario más la costra, influyen sobre la chapa $y$ reciprocamente, como justificación de hablar ante ustedes de la upiely del hormo, en lugar de solo de uno de sus componentes como podría correctamente deducirse del titulo con que anuncié esta colaboración mía, a sus trabajos, en esta II Reurión de la Sociedad Espa nola de Cerámica.

Y paso a ocuparme de las condiciones que cada parte de la upielw ha de satisfacer para llenar su cometido.

\section{El turber de chape}

El horno rotatorio es una viga continua que gira. Se sustenta en pares de rodadura constituzídos, generalmente, por un aro y dos rodillos de apoyo. Los espesores de la chapa, a lo largo del horno, son dimenslonados, en general, con coeficientes de seguridad, cada dfa más elevados, para quedar a cubterto de imprevistbles desviaciones de funcionamiento con relación a la marcha supuesta en el proyecto de construceión. Ios hornos de cemento, en general, son ampliamente resistentes a las solicltaciones por flexión, por esfuerzo cortante y por torsion. No puede decirse lo mismo de las deformaciones de su sección transversal. La forma de equilibrio de una cualquiera no es el círculo pretendido en su construccion, sino una elipse modiflcada en los apoyos (fig. 3) de modo profundo, segín Boh. man (3). 


$$
0
$$




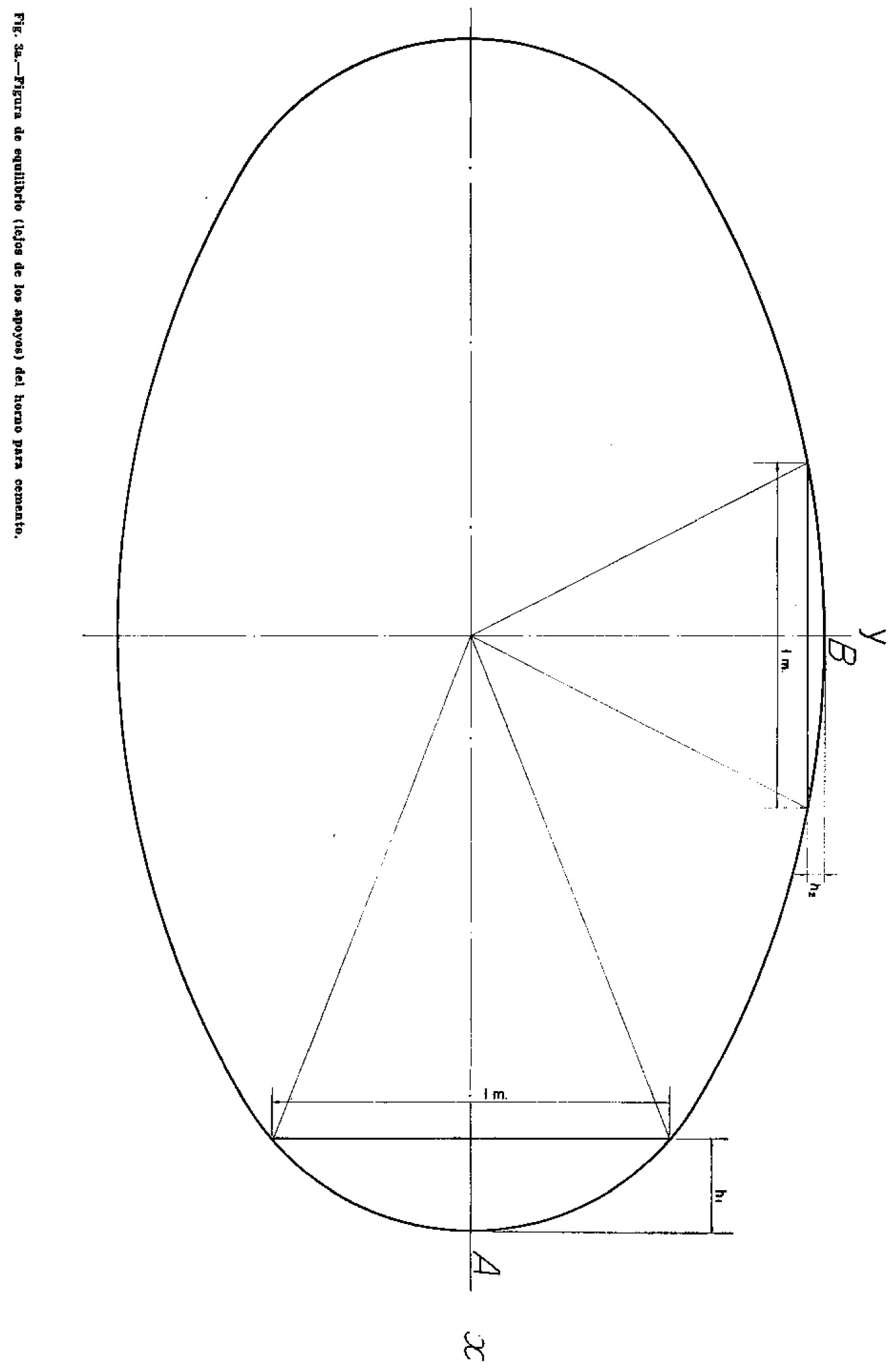

(c) Consejo Superior de Investigaciones Científicas Licencia Creative Commons 3.0 España (by-nc)

http://materconstrucc.revistas.csic.es 
La desviación de forma de la sección, con relación al ćrculo imaginado en la construcción del horno, puede expresarse por la magnitud denominada wovalidads (en $\mathrm{mm}$ ):

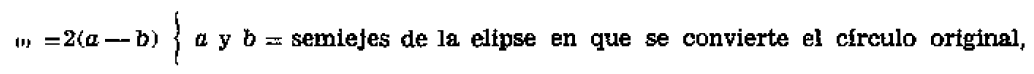

la cual puede determinarse experimentalmente de modo cómodo, mediante un dispositivo llamado Shelltest, fabricado por Skanska Cemenstaktiebrolaget.

El Shelltest es un compás de curvatura que define, entre puntas, una cuerda de un metro. Un tornillo situado en el punto medio del aparato permite medir, por su desplazamiento, las variaciones de la flecha de los distintos arcos abarcados en la curva intersección del plano de s.metrfa longitudinal del aparato, con la superflcie en que el eparato se apoya.

Si llamamos

$$
\delta=h_{1}-h_{y}
$$

a la desviacion máxima de las lecturas obtenidas al fijar el aparato sobre la chapa en un plano normal al eje del horno (fig. 3a), haciendo observaciones durante ura vuelta (el shelltest se fabrica para funcionamlento y registro automático), Rosenblad (4) obtiene la relación

$$
\text { (1) }=\frac{4}{3} d^{3} \cdot \delta \text {, siendo } d=\text { ciámetro libre del horno, en metros, }
$$

que permite deducir la ovalidad en cualquier sección normal al eje del horno, a partir de la máxima desviación hallada en una vuelta de aquel, y establece la dependencia entre ovalidad, deformación leida con el Shelltest $\mathrm{y}$ diametro del horno.

Este dispositivo para medir deformaciones del tubo del horno fue realizado por Skanska Cementaktiebolaget, preeisamente para tratar de adquirir ideas netas, dentro del complicadísimo campo de la duración de los refractarlos, en las fäbricas del grupo, así como las causas que la limitan. Se trataba de descubrlr si, entre deformación y duración del refractario, había o no una correspondencla, digamos, blunfroca.

Los resultados no han sido concluyentes, pero si lo suficientemente interesantes, sin embargo, para haber llegado a establecer clertas conclusiones acerca del temá que hoy nos ocupa, a la vez que proporcionar datos para la tecno logía de fabricación de homos (disposictón de los pares ce apoyo y rodadura, forma de la sección de los aros de apoyo, $y$ fijación de éstos al tubo rotatorio).

Recientemente, H. Huber (5) ha publicado, en forma r ráfica (fig. 4), los resultados estadísticos de las observaciones realizadas con el Shelltest, orientados a dar idea de la dependencia entre ovalidad y duración del refractario, para buenas y malas condiciones de la costra. El autor hace constar que está convencido de que sul litento puede parecer atrevido y que darí lugar a muchas discusiones. Señala, también, que los restultados se refieren al vestido refractario de la wzonas con ladrillos de magnesita, de espesor inicial de $180 \mathrm{~mm}$, fabricados por vía ignea o me diante aglomerante químico y colocados en hornos que trabajan por via húmeda o seca. Los datos de partida para la flgura tienen una antigtiedad de tres años. Nueva información complementaria, recogida desde entonces, segín el autor, parece que permite afrmar que existe correlación entre deformación de la chapa y duración de refractario, dentro de una udispersion" técnicamente aceptable.

Como orientación, Hilber, en su trabajo, establece que, para buenas condiciones de formación de costra, la uovelioads puede llegar a $20 \mathrm{~mm}$ si el diámetro está entre 3-3.50 metros. Con malas condiciones de mantenimlento de la costra (p. e., destrucción del ladtillo por derrumbamiento de trozos de aquélla, destrucción de la misma por defectuosa conducción del horno u originada por trabajo excesivo de la chapa), la ovalldad no debe pasar de 5 a 10 mm. Diámetros de horno mayores exigen ovalidades más pequefias.

La consideración de la ovalidad de la sección del horno nos ha llevado a darnos una razón más, a nuestro juicio en la limitación práctica de la altura de los ladrillos del vestido de los hornos, limitación aceptada por los cementeros y hasta recogida en algunas normas oflciales.

La figura 5 contiene, graficamente, nuestro intento de explicación.

La deformación del tubo implica la tendencia a penetrar cada dovela en la contigua, y las expresiones de correlactón entre deformación, modulo elóstico del material refractario e intensidad de la acción creada por la deformacíon, equilibrada solamente por la resistencia que aquél ofrezca, levan a la conclusión de que la acción es proporcionai a la altura de la dovela. Esta acción tiende a acortarlas por la proximidad a la cara ofrecida al interior del hormo, con intensidad proporcional a su altura.

La acción serx́ tanto más maniflesta cuanto mayor sea la ovaiidad adquirida por Ia sección transversal y que, seguin lo visto anteriormente, es función creciente con el cuadrado del diámetro del homo.

\section{Referactorin}

El refractario, que en general se dispone directamente sobre la chapa, ha de cumplir las siguientes funciones:

1. Aislar térmicamente el recinto interior del horno del medio ambiente, a través de la chapa, buena conductora det calor. 

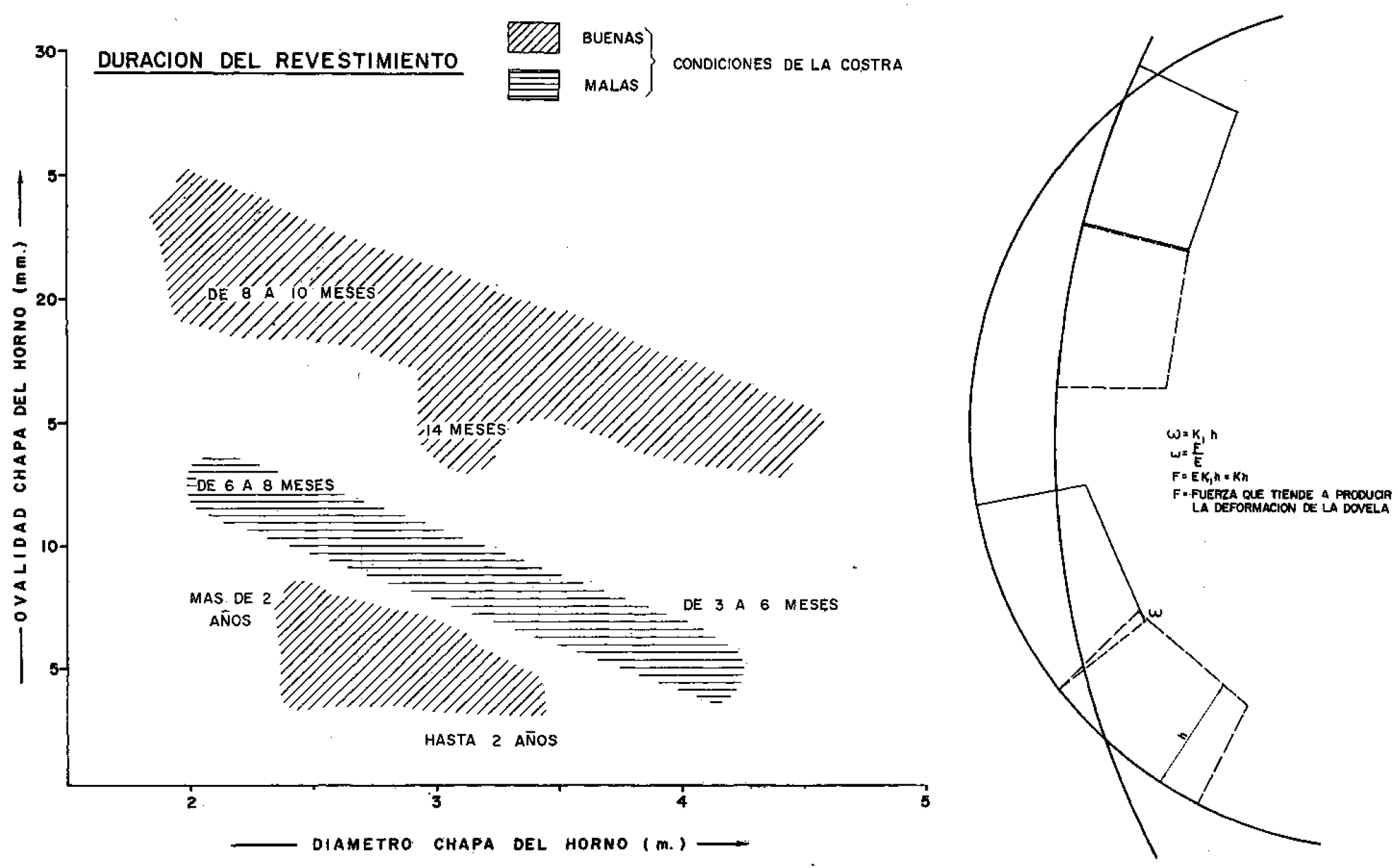

Fig. 4. Diámetros, deformación transversal de la secelón del horno y costra.

Fìg. 5.-Allura de dovela y detormación del horao. 
2. Constituir un buen stibstrato para la iniciación de la formación de costra y su conservacion en la zona donde aquélla se establece (zona de reacción y clinkerización).

3. Permitir al horno una actividad con el mayor intervalo de tiempo posible y con un espesor tal que aé lugar a un gradiente de temperatura que haga que la de la chapa no alcance valores inadmisibles, para su integridad (aparición de la urosa» o uroseta», equivalente a incandescencia de aquella, en léxico cementero).

Los gradientes térmicos, a lo largo del horno, para los gases, material en reacción y refractario o refractario ruás costra, suministran un criterio útil para determinar las directrices de preparación de un vestido para hornos de cemento.

Las zonas de secado y deshidratación no requieren otras condiciones que las que suele ofrecer un buen ladrillo cerámico, bien cocido y duro, para hacer frente al trabajo de rozamiento por el polvo o por la pasta $y$ las cadenas (via húmeda).

La zona de calcinación requiere ya un material de confección más cuidada (temperatura del orden de $1.000^{\circ} \mathrm{C}$ ), tratado térmicamente, de modo tal, que ya no puedan aparecer contracciones ulteriores.

Su duración, generalmente, es muy dilatada como en el caso de la zona de secado y deshidratación, pues no sufre de modo apreciable efectos de agresión química por razćn de no aparecer en el crudo, en esa región del horno, fase líquida ni ternperatura suficientemente elevada que pueda dar lugar a reacciones en estado sólicio, entre crudo y ladrillo, de velocidad medible.

En estas regiones del horno suele acentuarse el aislamiento térmico ofrecido por el revestimiento refractario propiarnente dicho, interponiendo, entre él y la chapa, un forro de poco espesor caracteristicamente aislante (ladrillos ligeros muy absorbentes), o błen aplicando, como vestido para esa zona, ladrłllo cerámico fabricado en condiciones tales que presente gran cantidad de htuecos en su masa para lograr una elevación de sti poder aislante. Con ello se consigue limitar la perdida, en cierta cuantia, del calor que pasa a través de la upiel" del horno.

El vestido de la zona de enfriamiento ha de presentar, como diferenciacion particular, gran resistencia a la abrasión, solieitación especifica de esa región del horno, por razón de la dureza del clinker (la influencia de la llama no es directa en esta zona). En esta región del homo, como en las de secado, deshidratación y calcinación, no parece justifcada la nota principai que implica el vocablo "refractario», que aplicamos generalmente al vestıdo de un horno de cemento.

En la zona de clinkertzación y reacción las solicitactones de origen térmico sobre el vestido del homo son las más rigurosas, pues en ellas se alcanzan las temperaturas más elevadas. Como las demás regiones a lo largo del eje del horno rotatorio, la kzonan sufre-como ya dijimos-la acción permanente de variaciones periódicas de temperatura (una por vuelta), de cuantia dada por la diferencia de temperatura de la pared del horno y del crudo en el momento de ponerse en contacto. Esto representa una exigencia que ha de llenar el refractario: ha de ser resistente al wchoque térmico», cuyo efecto, si el material no es apto, se tradıce en udesconchamiento» de la pieza.

La figura 1 permite obtener una orientación del valor del salto de temperatura entre pared $\mathrm{y}$ erudo, por consideración de los gradientes a lo largo del horno.

Por lo tanto, el vestido de esta zona, a la que antes llamé crisol o ulaboratorio la cual anvueive a la llama del mechero, adquiere temperaturas que ya justifican que el material de que haya de estar formado se le llame arefractarion. Además, Ios ladrillos que constituyen el vestido tienen que conservar la integridad de su forma, sornetidos a los esfuerzos que supone aguantar su peso propio, el del materłal que sobre él rueda y avanza y-como apuntamos-los engendrados por la deformación transversal del tubo y por su trabajo mecánico como viga.

La propledad de resistencia a los esfuerzos de compresión, en calfente, exigida a los ladrillos, se mide por la temperatura a que se inieia su deformación plástica, que se la designa como reblandecimiento bajo carga.

A las exigencias punto de fusión y reblandecimiento bajo carga hay que añadir otra de carácter especifico en la fabricación de cemento Portland: el vestido de la zona de reacción y clinkertzación. El arefractario" por antonoma sia, en plática de cementeros, ha de ser resistente a la agresión quimica del clíniker, en periodo de formación y en régimen de avance a lo largo del horno, a temperatura del orden de $1.500^{\circ} \mathrm{C}$.

Ante esta exigencia para el vestido de la kzonas, la refractariedad y el poder aislante, siendo condiciones imprescindibles, se convierten en complementarias de la propledad de ser prácticamente inerte, de reactividad, digamos nula, ante la presencia de una masa fuertemente básica, a temperatura muy elevada. La composición fisico-química del material que forme el vestido, ha de ser, de modo esencial, la adecuada a la consecución de esa resistencia a la agresión.

La agresión sobre el refractario es sostenida y facilitada por la presencia de fase líquida, cuya importancia y mecanismo de acción nos han sido expuestos, de modo magistral, por el señor Sorta en su conferencia. Por ello, se nalamos una caracteristica más a las esquematizacias anteriormente; por to tanto, el material para el vestido de la nalamos una caracteristica más a las esquematizadas anteriormente; por lo tanto, el materiai para el vestido de la zona, debe ser denso, con sacrificio de su conductividad termica y de su resistencia al choque térmico (variaciones
bruscas de temperaturas) para ofrecer la máxima dificultad posible a ia penetración, en su seno, del liquido engendrado (también a los productos gaseosos, gases de combustión y álcalis) durante el proceso de clinkerizaclón.

Los materlales empleados en la confección de las piezas que han de formar el vestido han de ser tratados térmi camente, como ya he indicado antes, hasta consegutir su estabilidad ulterior de volumen, a la temperatura de tra bajo en el lugar del horno en que se hallen. El doctor Alvarez Estrada, en su interesantísima conferencia dio razones y adujo pruebas experimentales de la importancia de tal estabilizacion, cuando nos informaba de la influencia de la procedencia mineralogica de los matertales para refractarios sobre las propiedades de éstos.

Suelen emplearse materiales diversos, todos ellos de elevada refractariedad como caracteristica común, y todos de un grado de resistencia a la agresion variable, pero siempre de elevado valor también. 
Estimamos mily difícil el establecimiento de un criterio de elección seguro, para preparar un material refrac tario para cemento. A las diferentes condiciones físico-quimicas conseguibles (en los minerales de partida) que im. plican ya una incertidumbre, digamos inicial, se suman las que suponen las condiciones físico-químicas de los crudos, cuya diversidad es, puede decirse, tan amplia como la que supone el número de fábricas en actividad, a lo que hay que añadir la influencia de las cenizas del carbón empleado, el westilon distinto en la conducción de los hornos, etcétera.

Por ello woy a limitarme a exponer ante ustedes, algo asi como la visión estadistica de los materiales que el fabricante de cemento tiene en cuenta cuando se ocupa del refractario de la kzonan.

La industria de cerámica refractaria, nacional y extranjera, refiriéndose a refractarios básicos, aplicables a la del cemento, of rece:

\section{Ladrillos silico-aluminnans}

Que por sus propiedades permiten intentar una ciasificación asi:

1. a) Ladrillos de areilla refractaria.-Obtenidos con productos naturales, ricos en caolinita. Su riqueza en alúmina no puede estar por encima del $46 \%$ (correspondiente a la caolinita deshidratada). Se reblandecen alrededor de $1.300^{\circ} \mathrm{C}$. Se consiguen ciertas modificaciones en sus propiedades (dilatación termica, densided, resistencia al choque térmico) variando tamaño de grano, elaboración do las plezas y tempergtura de cocción.

1. b) Ladrillos con contenido entre 46 y $\%$ \% de $\mathrm{Al}_{3} \mathrm{O}_{3}$ - Pueden constderarse, digamos, como si fueran derivados del mineral liemado silimanita [ $\mathrm{SiO},(27,7 \%) \mathrm{Al}_{2} \mathrm{O}_{3}(72,3 \%)$ ], y en su fabricación se emplean, a veces, efectivamente (según nuestra información), minerajes de este grupo-andalucita y cianita (la silimanita es muy $t 5$ casa)-. Incluyo en este personal criterio de clasifeación, hasta un contenido de alúmina proximo a $70 \%$.

1. c) Ladrillos de alto contenido de aluimina (de 70 a $100 \%$ ). - Parece que suelen fabricarse con grancillas de mullita pura $\left[3 \mathrm{Al}_{2} \mathrm{O}_{3}(72 \%) ; 2 \mathrm{SiO}_{2}(28 \%)\right]$, conseguida a partir de bauxitas ricas, $y$ también con corindones naturales, obtenidos artificialmente en el horno eléctrieo (1.400 y $1.600^{\circ} \mathrm{C}$ ). Su refractariedad (de 2.750 a $2.000^{\circ} \mathrm{C}$ ) $\mathrm{y}$ temperaturas de reblandecimiento bajo carga (de 1.450 a 1.600 ) crecen monotonamente con el contenido de alúmina.

Su densidad es correlativa también con el contenido en alúmina, influida, a su vez, por la porosidad, aunque se observe, por los datos de que disponemos, que éste alcanza valores muy comparables, consecuencia probable de la atención prestada, a este aspecto, por la tecnología de la fabricación de refractarios.

La resistencia mecánica a compresión es más alta también a medida que crece el contenido en alúmina y el es mero de su fabricación.

Para la resistencia al choque térmico encontramos datos relativamente comparables para toda la gama de riqueza en altimina; lo mismo parece deducirse para la dilateción a $1.000^{\circ} \mathrm{C}$ y la contracción residual permanente, cuya importancia creemos que debe de estar muy influida por las condiciones de cocción y enfriamiento de los ladrillos.

Para la resistencia a la agresión de los ladrillos ślico-aluminosos, por el material presente en la zona de cocción, no encontramos en la bibllograffa, en general, más que calificaciones de buena, ete, que carecen de valor positivo, por su ausencla de carácter cuantitativo (cuyo establecimiento nos parece, desde luego, de la mayor dificultad) en relación a la duración previsible, ante las solicitaciones implicadas por su aplicacion al horno para cemento.

Mi experiencia personal sólo me permite afirmar ugrosso modon, que la resistencia a la agresión (para mi deci. siva, en la duración del refractario en la azonas del homo) crece con el contenido en alúmina, aunque suscriba las observaciones citadas por los señores Alvarez Estrada y Soria Santamaria, ratificando que aquella correspondencia entre contenido de alúmina y duractón del refractario no puede tomarse como decisiva.

Parece que la sola consideración de la participación gravimétrica de un componente, por esencial que pueda ser en la composiction químice de un refractario, si en algín sentido dice mucho no explica en absoluto todo en rela ción a la cuantía de la duración de aquél, aun para condiciones industriales comperables de normas de confecoión y tratamiento térmico aplicados.

Permitidme que traiga a esta conferencia una observación personal que parece afirmar lo que antecede, aunque sea consciente, por mi parte, de su pequeño valor informativo por lo reducido de los datos en que se apoya y en no haber podido, hasta ahora, realizar un ensayo sistematizado que confirmara o negara lo que parece deducirse de ellos.

Dificultades de abastecimiento de refractario de kzonan, en el año 1946 (recién iniciada mi actividad de trabajador al servicio del cemento), me llevaron a aplicar en el horno ladrillos con un contenido de $\mathrm{Al}_{2} \mathrm{O}_{2}$ del orden de $45 \%$ con un resultado desastroso, pues duraban alrededor de veinte dias.

Los datos archivados en ta f́b́brica, acerca de la composición de refractario y su duración, me llevaron a la consideración de que la presencta de Fe $\mathrm{O}$ en $\mathrm{los}$ ladrillos de alta proporción favorece la resistencia de los mismos a la agresión por el crudo, aigo así como si, a tal efecto $\mathrm{Al}_{2} \mathrm{O}_{3}$ y $\mathrm{Fe}_{2} \mathrm{O}_{3}$ presentaran clerta capacidad de sustitución reciproca.

Siguiendo ideas, indudablemente muy simplicistas, me postulé que en un refractario a alta temperatura todo to

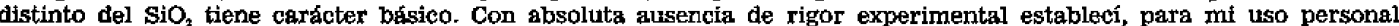


un índice en el que pretendía orientar mi juicio, wa priori», acerca del valor eficaz de un refractario, cuando, además de la alúmina contuviera una dosis de $\mathrm{Fe}_{2} \mathrm{O}_{n}$ de cuantia blasfematoria a los efectos del concepto arefractariedad".

Tal indice, que denominé de acidez del refractario, tiene por expresión:

$$
\mathrm{N}=\frac{\mathrm{SiO}_{2}(\%)}{0,37 \mathrm{Al}_{2} \mathrm{O}_{\%}(\%)+0,97 \mathrm{Fe}_{2} \mathrm{O}_{\mathrm{g}}(\%)+1,07 \mathrm{CaO}(\%)+1,5 \mathrm{MgO}(\%)}
$$

Esto implica aceptar-insisto que sin justificación experimental alguna-la aparición de mullita, hipersteno, wollastonita y enstatita, como resultado de la combinación total de $\mathrm{Al}_{2} \mathrm{O}_{3}, \mathrm{Fe}_{2} \mathrm{O}_{3}, \mathrm{CaO}$ y $\mathrm{MgO}$ con la $\mathrm{SiO}$.. Es superfluo decir que, si el indfee es inferior a 1 , el ladrillo con esta definición será básico, tanto más cuanto más se separa de aquel valor, y ácldo, si es supertor a 1. La existencia de los compuestos en que se apoyan los coeficientes de los óxidos básicos es-como sabemos-problemática.

Orientados por esta idea y ante la penuria, al parecer difficil de salvar, de adquisición de bauxitas en España, solicité la fabricación de ladrillos con contenido en $\mathrm{Fe}_{2} \mathrm{O}_{3}$ del orden del $10-15 \%$.

Seguimos con interés su duración y los resultados obtenidos, tomando como variable independiente el indice $\mathrm{N}$, los reproduce la fig. 6 , en la que hemos indicado algunos puntos representativos, señalando el contenido en $\%$ de $\mathrm{ALO}_{3} \mathrm{Y} \mathrm{Fe}_{2} \mathrm{O}_{3}$

Afortunadamente, han pasado los años de penuria de materias primas para la tecnologia de producción, en confunto $\mathrm{y}$, por tanto, ha pasado la actualidad de soluciones más o menos heroicas y también, quizás, la de mis intentos de mediclón de hasta dónde es posible llegar con las adiciones y sus resultadós a los efectos de la agresivi. dad del refractario.

Quede ante ustedes, como muestra de mi preocupación por este tema, y como pública afirmación de la paciencia de un fabricante de refractario muy estimado.

En los ladrillos ślico-aluminosos, hasta muy alto contenido de $\mathrm{Al}, \mathrm{O}_{\text {, }}$, la mullita es el componente principal $y$ responsable de las cualidades refractarias, bajos coefictentes de dilatación y estabilidad al choque térmico de aquéllos.

La formación de mullita es, esencialmente, una reacción en estado sólido dependiente, por tanto, de modo esencial, de la temperatura (del orden de $1.500^{\circ} \mathrm{C}$ ), que depende, a su vez, de las propiedades fisicas y de la composicíon material de los productos de partida.

La mullita, por calefacción muy prolongada, se descompone en corindón y en una fase vitrea que contiene silice sctiva libertada.

Por ello, en la gama de fabricación de ladrillos silico-aluminosos, especiammente en los de alta alumina, encontramos aplicaciones variadas de adictones que neutralicen la siltce liberada en el proceso de mullitización, siltice que limita, a veces, si no se combina (por las propledades que imparte al refractario) el campo de aplicación de éste.

Se practican, con tal fin, adiciones de bauxitas muy puras, asl como de óxidos de cromo, circonio, calcio, mag. nesio e, incluso, dolomita. De entre todos se ha encontrado, como más conventente, el oxido de magnesio, porque con el la $\mathrm{SiO}_{2}$ amulííican lfbre queda eliminada en forma de forsterita; Inciuso con exceso de magnesia, resulta un conjunto de punto de fusión más alto. Esta posibilidad de absorción de $\mathrm{SiO}_{2}$ naciente, reactiva, ha dado lugar, incluso, a procesos de elaboración de refractarios silico-aluminoso-magnesianos, a los que tambiến haremos una rápida referencia.

\section{Ladrillos a base die magnesita, de dolomita sulfeo-umagnesianos:}

No tengo experiencia de trabajo personal con refractarios de este tipo. Os voy a ofrecer la informacion que mis posibilidades y mi interés, por un lado, y el escaso tiempo libre y mi tarea diarla, por otro lado, me permiten.

Lo hago con el fin de que, quizás por razón de mi carácter de trabajador del cemento, algo de mi pequeña información tuviera la fortuna de ser noticia para vosotros, en relación con vuestras producciones para nuestra actividad.

Se ha invertido gran cantidad de trabajo científico para explicar las razones de utilización y las propledades refractarias y de inercia químies de Ia magnesita y de otros productos en cuya constítución entra aquel óxido. Como de la mullita y del corindón, de este oxido son conocidas todas las notas físico-químicas que permiten operar con y sobre esta familia de refractarios con maestria.

Tambiẹn hay descripciones detaliadas de sus procesos de fabrlcación en libros y revistas, etc.

Como antes, me limitaré a considerar, desde el punto de vista del usuario, la gama de productos que es ofrecida a su demanda.

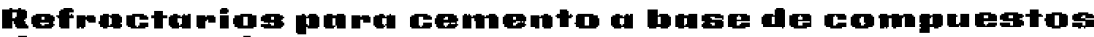 de magnesio}

La magnesita y la dolomita han tomado carta de naturaleza entre las materias primas para refractarios en el campo de la siderurgia, con el descubrimiento en 18\%9, por Thomas Gilchrist, de su capacidad de combinación del fósforo en los convertidores. 


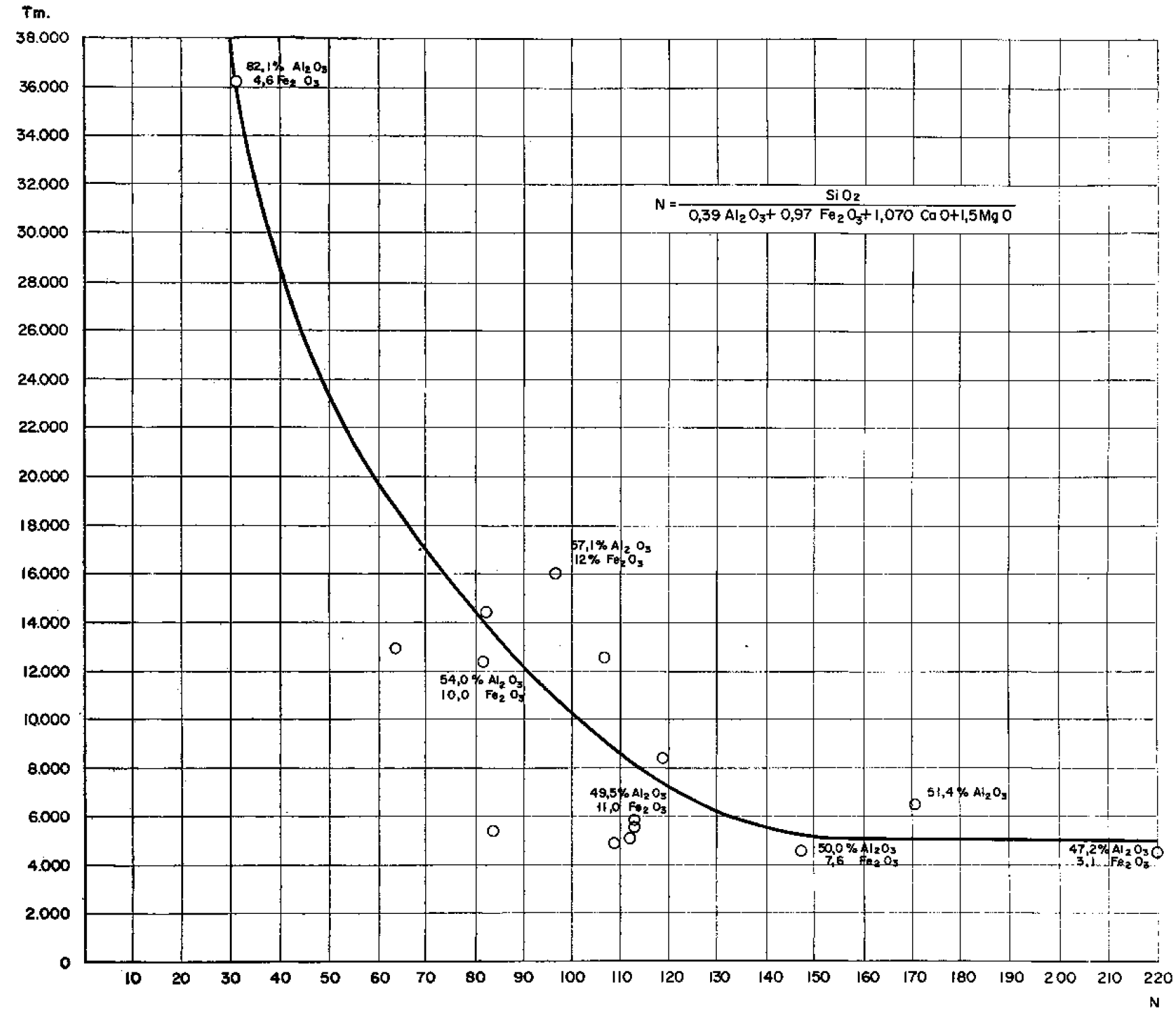

Fig. 6.-Duración del refractario en función del findice N. 
Hoy se aplican en ditersos ramos de la producción, en particular la magnesita, y de modo creciente en la industria del cemento, especialmente en Europa.

Recientemente, se han introducido, con el mismo caracter de materias primas para refractarios, algunos siliçatos de mngnesio (forsterita y cordierita), y los derivados de la aplicación de forsterita han encontrado campo de apli cación en los revestimientos para el horno de cemento.

\section{Ladrillos de magnesita}

En su preparación se parte de la magnesita (giobertita) amorfa, producto de meteorización de rocas silico-mag. nesianas (olivino y serpentina). Menos frecuente de espato magnésico, roca cristalina de origen metamorfico por reacción del araconto (carbonato ćlicico) y de kieserita (sulfato magnésico). Las propiedades de ambos carbonatos de magnesio, implican sólo pequehas diferencias en el proceso de preparación de plezes refractarias, pues la magnesita espática cede su carbónico más lentamente que la amorfa.

Nos parece de interés citar que, cuando hay dificultades de disponer de yacimientos adecuados, se han propuesto y utilizado métodos de recuperación de $\mathrm{MgO}$ a partir de las lejías finales en la elaboración de la carnalita, por ejem plo. $Y$ también se emplea, quizés con otros fines (obtención del metal), la extracción de Mgo a partír del agua del mar.

La magnesita ha de ser descompuesta por la acción del calor, llegando hasta la temperatura de sinterización la cua viene influida por las impurezas (fundentes) que acompañan a la magnesita natural. In sinterización implica un notable crecimiento de los cristales de periclesa (MgO cristalizado) con aumento de densidad; esta magnitud sirve para definir la aptitud de empleo en los refractarios (eliminación de ulteriores contracciones durante su utilización). También se apllca, a veces, magnesia fundida en el horno eléctrico. La temperatura de sinterización de la magnesita natural llega a $1.600-1.700^{\circ} \mathrm{C}$. Condiciones de compostelón adecuada (impurezas) la rebajan hasta $1.500^{\circ} \mathrm{C}$.

La elevada refractarledad de la periclasa $\left(2.800^{\circ} \mathrm{C}\right)$ ofrece un margen de amplitud tal, que aquella propiedad no defne, por decir asi, a los refractarios de magnesita. La temperatura de rebiandecimiento y la estabilidad son, por ello, la referencia de bondad de los Ladrillos.

Ambas propledades son influenciadas deswentajosamente por la silice, aunque su presencia, en clerta cuantía, apoya la cristalización de la periclasa.

También la cal presente en la masa de los ladrillos es perfudicial, si su contenido excede de clertos límites en el conjunto de los fundentes.

Para hacer inactiva esta cal se agrega alúmina pura, que, por intermedio de la presencla de aluminato magnésico, da lugar a aluminato cálcico no perjudicial.

Los productos bien elaborados ofrecidos por la industria de los refractarios ofrecen al cementero las siguientes

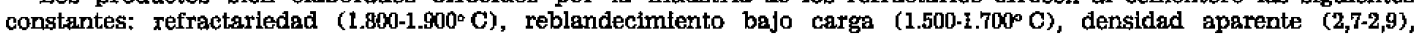
porosidad $(19-22 \%)$, contracción residual $(0,1-0,3 \%)$, resistencia al choque térmico (25-27 clelos)

La resistencia a la agresion es, cualitativamente, muy alta, afirmación basada en la dilatada duraclón de los misrnos en el horno en explotación. En el mercado existen abundantes productos, respaldados por flrmas evidentemente solventes.

\section{Laderiture de dollonetita}

La dolomita se aplica como materia prima en la industria del refractario. Como es sabido, se trata de un carbonato doble de calcio y magnesio. En los materiales dolomíticos, el contenido en ambos varia entre limites muy amplios. Como materia prima se aplica la dolomita normal, que contiene cal y magnesia en cantidades equivalentes o las más ricas en magnesia, producto de descomposición de la dolomita normal.

La presencia de cal en gran cantidad constituye una diflcultad para la aplicación de la dolomita calcinada y sluterrizada, por su reactividad a los agentes atmosféricos, djficiltad salvada por impregnación de la dolomita sinterizada en alquitrán, que, a su vez, sirve para dar la posibilioad ulterior de formar los fadrillos.

Tambien se ha aplicado el recurso de agregar alúming, como indicamos al tratar de los ladrillos de magnesita y aun otros óxidos, tendentes a eliminar la cal utilizando las distintas temperaturas de caustlficación de los carbonatos.

De aplicación en la industria metalúrgica, no tenemos noticia confirmada de la introduceion de ladrllos de dolomita en la industria del refractario para el cemento y, la citamos solamente como informactón de tipo general.

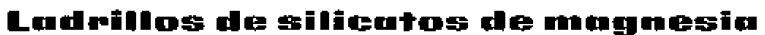

De Ios silicatos de magnesia son aplicables, por sus constantes físico-quimicas, solamente el ortosilicato, puesto que, al lado de su elevado punto de fusión $\left(1.910^{\circ} \mathrm{C}\right)$, muestra otras excelentes propiedades como material refractario. Fn la naturaleza los ortosilicatos de megnesio están representados por la forsterita (SiO $2 \mathrm{MrO}$ ), poco frecuente, y por el olivino (mezcla isomorfa de forsterita y fayalita ( $\mathrm{SiO}$. 2FeO), mucho más aburidante. Como el olivino funy por el olivino (mezcla isomorfa de forsterita y fayalita (SiO.. 2FeO), mucho más abundante. Como el olivino fun.
de a $1.557^{\circ} \mathrm{C}$, solamente queda la forsterita como material natural apto para la obtención directa de refractarios.

sin embarco, debido a su escasez, se han ideado diversas soluciones para conseguir masas ricas en forsterita a partir de ollvino y de dunita y aun de serpentina (hidrosilicatos de magnesto, sustituida ésta parclalmente por hleTro). LA utilización de estos materiales supone siempre un contenido de hierro (en FeO) menor del $10 \%$. 
Como lo que se persigue es la obtención de forsterita, trabajando con estos silicatos, se agrega magnesita calci. nada (impuriffcada también por hierro), que reacclong con la silice libre que pudiera existir, dando forsterita e incorporando el hierro en forma de ferrito magnésico. Este esquerna simplista para llegar a la obteneion de forsterita a partir de olivino o sorpentina, ete. implica, sin embargo, una tecnologfa que, aunque dificil, ha tenido el aliciente sufloiente para ser puesta en explotación.

En el mercado existen ya ladrillos de forsterita, de los cuales hemos visto citadas las siguientes constantes: tem. peratura de fusión, $1.800^{\circ} \mathrm{C}$; temperatura de reblandecimiento bajo carga, $1.500-1.700^{\circ} \mathrm{C}$; densidad aparente, 2,80; conductividad térmica, 2,3; choque térmico, 25 .

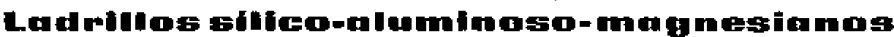

Ya hemos dicho anteriormente que la formación de mullita y corindón en el tratamiento térmico de los ladrillos sílico-aluminosos da lugar a la liberación de sílice activa, que, sin duda alguna, representa un elemento poco resis. tente a la agresión ante la solicitación del crudo de cemento.

También hemos señalado que esa silice se neutraliza por el intento de adiciones variadas, y que la de Mgo es la más adecuada por dar lugar a la presencia de forsterita.

Un ladrilio que tuviera, por una parte, forsterita y alúmina, por otra, daría lugar a un sistema final como el que describe la siguiente ecuación

$$
\mathrm{SiO}_{2} \cdot 2 \mathrm{MgO}+\mathrm{Al}_{2} \mathrm{O}_{3}+\mathrm{Al}_{2} \mathrm{O}_{3} \cdot \mathrm{MgO}+\mathrm{SiO}_{2} \cdot \mathrm{MgO}
$$

(espinela) (enstatita)

cuyo punto de fusión sería de $1.250^{\circ} \mathrm{C}$.

De modo parecido, por fusión de cantidades estequiométricas de $\mathrm{Al}_{2} \mathrm{O}_{3}$ y $\mathrm{MgO}$, para dar espinela, a las que se agregará una parte de ślice y dos partes de magnesia, se podría llegar a un ladrillo que al lado de la espinela (p. $f, 2,135^{\circ} \mathrm{C}$ ) contendria forsterita. También partiendo de caolín, fundido con forsterita, se puede obtener espinela enstatita ( $\mathrm{StO} . \mathrm{MgO}$ ) y silice libre, que puede ser absorblda con exceso de alumina pura o magnesita.

Los ladrillos a base de forsterita y espinela parecen ofrecer un vasto campo a las posibilidades de obtención de ladrillos de alta refractariedad $y$ con las valiosas propiedacies restantes exigjas por los aplicados a la industria cementera.

Existe ya en mercado algún producto que responde a esta idea. De él conocemos: p. de fusión, $1.990^{\circ} \mathrm{C}$; reblandecimiento bajo carga, $1.500^{\circ} \mathrm{C}$; densidad aparente, 2,7; resistencia al choque térmico, 27-40 ciclos.

Fl conocimiento de las constantes reticulares de la espinela, que por su rempaquetamientow densisimo de jones oxfgeno define su estabilldad especiflca, ha dado una base sólida en que apoyar la producción de refractarios básicos de alta calidad, de los que son ejemplo los de cromo-magnesita, introducidos, en modo amplio, en la siderurgia y citados, en ocasiones, en la del cemento.

Existen minerales naturales con estructura de espinela, entre los que figuran, aparte de la cromita, la hercynita $\left(\mathrm{FeO} . \mathrm{Al}_{2} \mathrm{O}_{3}\right.$ ), la magnetita ( $\mathrm{FeO}$. $\mathrm{Fe}_{2} \mathrm{O}_{3}$ ) y otros que por su estructura de espinela parecen ofrecer la coyuntura de llegar a ser considerados desde el punto de vista de fabricantes y usuarios de refractarios básicos. La titanomagnetita, con estructura de espinela inversa $\left[\left(\mathrm{FeO}\right.\right.$. $\left.\mathrm{TiO}_{2} \mathrm{Fe}_{2} \mathrm{O}_{3}\right]$, también ha debido de recibir la atención de los fabr:cantes.

\section{Ladrillos especiales}

El mercado alemán ofrece ladillos especialmente fabricados para resistir a la abrasión del clínker a la sallda del horno, $\mathrm{y}$ ladrillos anti-costra, basado en la aplicación del oxido de titanio con puntos de fusión alto (1.840 $\mathrm{C}$ ) y de reblandecimiento bajo carga de $1.625^{\circ} C_{\text {; }}$ densidad aparente de 3,1

\section{Ladeillos aiglamtes}

Con conductividad térmica, del orden de 0,20 cuando son muy poco resistentes a la compresion (del orden de $\left.7-9 \mathrm{~kg} / \mathrm{cm}^{2}\right)$ y conductividad más alta $(0,42)$, pero más resistente $\left(40-100 \mathrm{~kg} / \mathrm{cm}^{2}\right)$, se obtienen partlendo de masas ceramicas cocidas con gran porosidad $(48 \%)$ y densidades aparentes del orden de $I$.

He pretendido dar a ustedes mi visión personal en lo más seliente de los productos ofrecidos al cementero, por la actividad de ustedes. Visión, estoy seguro que incompleta, aunque cada día la estimo más interesante para mí. Ia tecnologia de la construcción, con sus requerimientos de materiales permanentemente mejorados que paranticen su empleo con conficientes de trabajo muy elevados, y la economía de producción de las fábricas de cemento mismas, por no citar mís factores, colnciden hoy, más que en cualquier otra epoca pasada, en llevar al Director de famas, por no citar más factores, colnciden hoj, más que en cualquier otra epoca pasada, en llevar al Director de fá-
brica de cemento al intento de alargar la actividad ininterrumpida de los hornos. $Y$ en esto, a consecuencia de los demés aspectos de la upiel» de aquellos, juega un gran papel haber acertado con el refractario más adecuado; de ahi, el interés del conocimiento tan amplio como le sea posible de lo que ustedes producen para el cementero. 


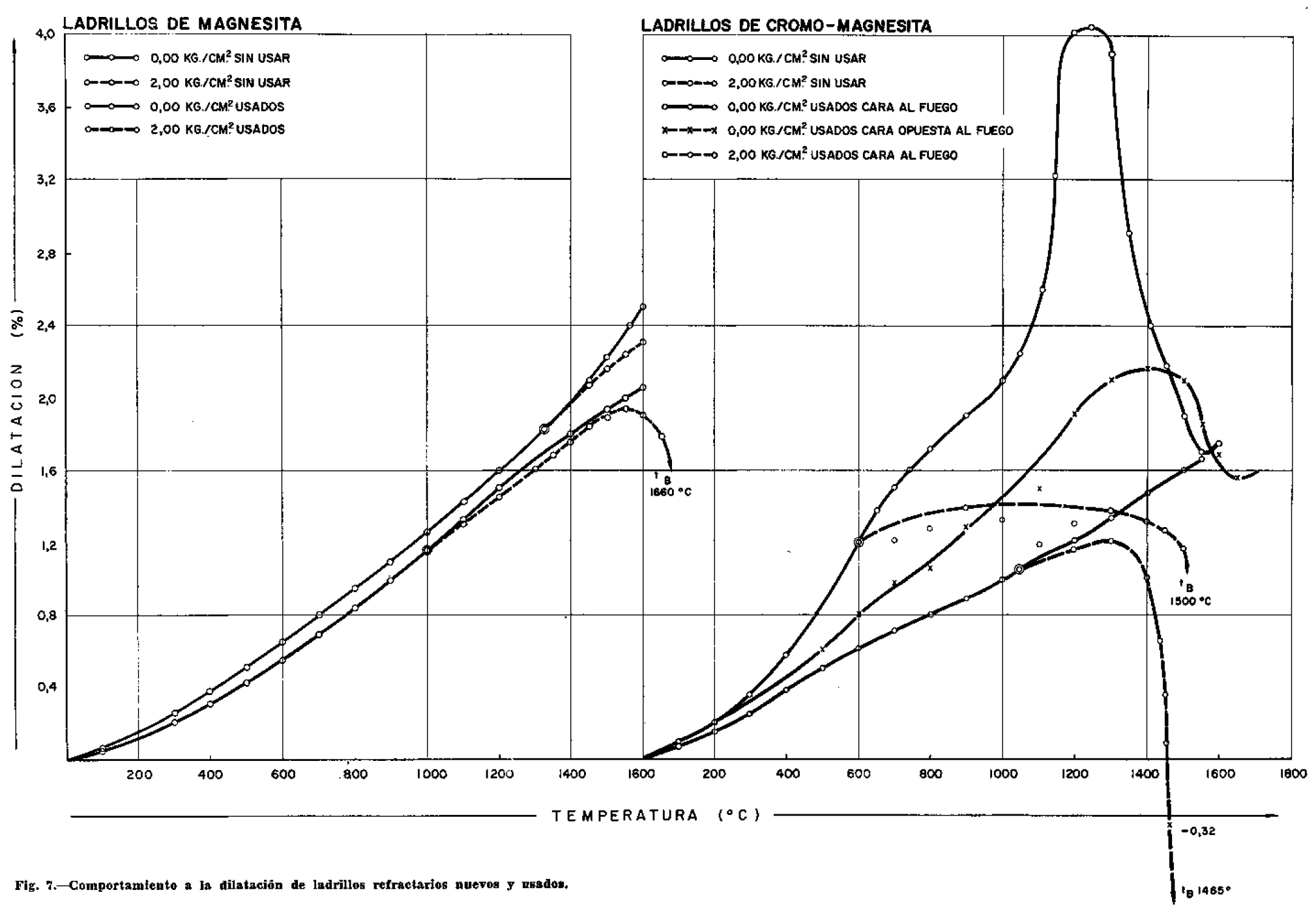

5 
Para tal conocimiento nos proporcionan ustedes datos interesantisimos, con la perfección y simpiicidad de los expresables en cifras, en relación con las constantes habitualmente llamadas fisicas y también las correspondientes a la composición material (análisis químico) de sus producciones. Sin embargo, yo echo de menos cuanta amplitudi fuera alcanzable, de lo que podernos esperar de un refractario recibido u ofertado ante la agresión de nuestro crudo, cuando entren en contacto en el horno.

Un dato que expresará la resultante global, para una composición química dada del refractario, es la influencia que, a los efectos de la agresión química, a la temperatura de trabajo, pueden tener la procedencia de las materias primas, y la existencis real y cuantia relativa de los comronentes resistentes a la agresión (mullita, corindón, fors terita, espinelas); éstos son consecuencia de la composición material, sin duda, pero también del proceso de fabricación del material refractario que, desde luego, estimo muy difficil y complejo, pese a la ayuda que, como para nos otros cementeros, pueden signiflcar los hallazgos de la Cristaloquimica y de la Quimica de altas temperaturas, hoy cultivadas con intensidad más alta que nunca y que ya en nuestro país tiene representantes de la categoría del doctor Aleixandre, aqui actualmente.

Soy consciente de las dificultades que entraña conseguir las lineas generales de un ensayo que ha de abarcar tantas variables y también los de realización correcta de su medición, a lo que se ha de sumar, después, su interpretación; sin embargo, perdonadme que insista en la necesidad de intentarlo.

Vuestra maestria y vuestro entusiasmo harin encender también el entusiasmo en ayudaros, por parte de los Centros de Investigación del Patronato Juan de la Cierva, especialmente el Instituto Eduardo Torroja de la Construcción y del Cemento, y el Departamento de silicatos, y no dudo que, por parte de mis colegas cementeros, tendréis cuantos datos os puedan interesar para el mejor planteamtento del problema.

\section{La costrat o Incrustaction}

Paso, finalmente, a ocuparme del tercer estrato de ta epiels del horno de cemento. La costra o incrustación repre senta un elemento importantísimo en el comportamiento de la splels del horno por stu capacidad de protección del Iefractario frente a las solleltaciones de la llama y a la agresión por el crudo. A su vez, la presencia de una buena incrustación comunica rigidez a la sección transversal, ventaja que añadir a las anteriores.

Para subrayar más el valor de la costra, como parte importante en la duración del refractario y, por tanto, en ias hornadas de larga duración, quiero citar el capítulo que a ella dedica el señor Soria, en su magnifico trabajo exhaustivo titulado wMateriales refractarios para la industria del cementon (Instituto Eduardo Torroja de la Construcción y del Cemento. Monografía nuim. 165), al que remito a cuantos interese el tema en general. En la parte dedicada a la costra expone, con maestria, la teoria, ensayos realizados, etc., que intentan explicar el mecanismo de su formación y conservación en el proceso en adesequilibrion representado por el sistema refractario-crudo en fase de clinkerización.

Sólo puedo añadir, por el interés que pueda signifiear para ustedes su lectura, un trabajo reciente de $H$. $E$ Schwiete (6), del que reproduzco la figura 7, en la que, de modo gráflco, se express el comportamiento, a los efectos de la cillatación sin carga y bajo carga de los refractarios de magnesita y de cromo-magnesita, nuevos y usados.

Para los ladrillos de magnesita la dilatación discurre normalmente $\left(2 \%\right.$ para $\left.1.400^{\circ} \mathrm{C}\right)$. Sin carga no hay deformacíon hasta $1.600^{\circ} \mathrm{C}$; para carga de $2 \mathrm{~kg} / \mathrm{cm}^{2}$ se inicia la deformación a $1.400^{\circ} \mathrm{C}$, pero no aparece deformactón plástica hasta $1.600^{\circ} \mathrm{C}$

Ladrillos que habian sido usados en un horno para cemento presentan dilatación lineal menor, atribuible a una estructura más suelta. Bajo carga comienza la deformación de 1.000 a $1.100^{\circ} \mathrm{C}$, y a $1.500^{\circ} \mathrm{C}$ aparece la deformación plástica. A $1.600^{\circ} \mathrm{C}$ el ladrillo es destruído.

Para los de cromo-magnesita observan particularidades tales como un máximo de dilatación en la cara del ladrillo expuesta a la acción de la llarna, por estar impregnada de álcalis, así como una elevación de densidad aparente de 2,91 a 3,20 y un descenso de porosidad de $8,3 \%$.

En la publicación ya citada de A. Majdic y H. E. Schwiete (2) hay otro interesantísimo trabajo acerca de la formación de costra, en el que se trata el ferómeno basándose en conceptos de la física superficial (mojabilidad, ángulo de borde, energia superfictal, adherencia) y de la viscosidad, y sus conclusiones vienen a conflrmar las de Mussnug (7), apoyadas en resultados estadísticos de explotación cementera.

En este trabajo de Majdic y Schwiete se describe el aparato experimental utilizado que, a mi juicio, es sencillamente impreslonante.

Todo parece indicar que el problema de adquirir conocimientos profundos acerca de las relaciones crudo-costrarefractarlo para llegar a dominar la realización de una buena adherencia, constituye un tema de estudio muy interesante.

Con lo que antecede en esta exposicion doy por concluido mi intento de definir to que, tan repetidamente, he llamado "pieln del horno cara cemento, la estructura de sus elementos constitutivos y su interdependencia.

El hormo en funcionamiento significa un sistema mucho más complejo en relación con sl1 wpieln que to que yo, en esta exposicion, he podido explicarles.

Conducción del horno, temperatura, forma de la llama, velocidad de rotación, grado de llenado, homogeneldad del crudo, su composición, su reactividad, cenizas del carbón, etc., son otros tantos factores muy de tener en cuenta 
cuando el fabricante de cemento enjuicia el sistema costra-refractario-envolvente metálico del horno frente a su importancia en relacion a la rentabllidad del proceso productor cuya responsabilidad soporta.

El refractario juega, como creo haberos explicado, un importante papel en esa rentabilidad, que ha sido estudiada de modo conereto, entre otros, de modo profundo y exhaustivo, por R. Münk (8), y su importancta actual en la industria americana del cemento, por $\mathbf{K}$. Peukert (9), quienes exponen las diferenctas de características del revestimiento aplicado (tipos aplicables, aislamientos, piezas normalizadas, etc.), basados, fundamentaimente, en razones económicas (coste del calor, precios de mano de obra) últimas y decisivas de todo proceso productor de bienes materiales.

No quiero concluir sin hacer presente, como colaborador en una industrla cliente de la que representan ustedes, cuán beneficioso sería el establecimiento de una comunicación permanente entre ambos y a través de los organismos oficiales dedicados a la investigación cientiflca, cuando la complejidad del tema, que pudiera plantearse entre ambos, requiriese la movilización de medios que, por ser especializados para la investigación, están muy lejos de ser utillaje habltual de los laboratorios de los centros de producción.

La normalización de tipos de refractario, formato de las plezas, definición de estipulaciones de recepción, ela. boración de ensayos para establecerios, etc. serian los logros a conseguir de un trabajo en equipo, en el que los cementeros jugarian el papel de ver (con toda su dificultad) la realidad del comportamiento del refractario; a partir de ello, los cienticos se aplicarian a juzgar y orientar, con sus decisiones, el actuar de ustedes en la realización del adecuado revestimiento refractarlo para la industria del cernento.

$\mathrm{Y}$ quiero concluir, agradeciéndoles la gentil atención que han prestado a mi exposictón.

\section{bibliografía}

(1) M. A. Fourror: "La flamme de charbon pulverisé dans le four rotatif à ciment". Journées de la combustion des combustibles solides et pulverisés. 426 .

(2) A. MAJDIC y H. E. ScHwIEIx: Uber die Ansatzbildung im Drehofen. Zement-Kalk-Gips, 12, núm. 3, marzo de 1959 , pág. 89 .

(3) W. BoHмas: "Fragen der Konstruktion und Repałatur von Drehofen". Zement-Kalk-Gips, 7, núm. 11, noviembre 1954, pág. 409.

(4) G. RosskBt.AD: "Radiale Deformation der Drehofenmantel". Zement-Kalk-Gips, 7, núm. 4, abril 1954, pág. 130.

(5) H. HuBER: "Stabilitatsmessungen und Drehofenmanteln mit dem Shelltestgerăt". Zement-Kalk-Gips, 14, número 1, enero 1961, pág. 1.

(6) F. E. Schwrerr: "Zementchemische Arbeiten ans dem Institut ftir Gesteinkunde". Zement-Kalk-Gips, 14, número 9, septiembre 1961, pág. 400 .

(7) M. Mussndg: "Ansatzbildung im Zementdrehofen und Futterhaltbarkeit". Zement-Kalk-Gips, 1, núm. 3, julio 1948, pág. 41.

(8) R. Mttwr: "Die statistische Erfassung des Verbrachs an Drehofenausmauerungen". Zement-Kalk-Gips, 3 , número 12 , diciembre 1955 , pág. 439 .

(9) K. PENKERT: "Kostenprobleme und Wirtschaftlichkelt der feuerfesten Ausmauerungen in U.S.A.". Zement.Kalk-Gips, 14, núm. 8, agosto 1961, pág. 352. 